Renata Jakubczuk

Université Marie Curie-Skłodowska, Lublin, Pologne

http://dx.doi.org/10.18778/8088-896-8.21

\title{
MINORITÉ FRANCOPHONE DÉFAVORISÉE DANS LA DRAMATURGIE DE MARCEL DUBÉ ZONE (1953), FLORENCE (1957), UN SIMPLE SOLDAT (1958)
}

\section{Introduction}

« Le théâtre est le reflet d'un milieu et d'une époque en autant qu'il reflète un individu qui est intégré à son milieu, à son époque $\gg^{1}$ avoua Marcel Dubé dans une interview, en 1972. En lisant ses textes, surtout ceux qui précèdent cette constatation du dramaturge, on ne peut qu'approuver l'auteur québécois car les protagonistes auxquels il donne vie dans ses pièces, dans son théâtre, représentent parfaitement la minorité francophone au Canada. Une minorité défavorisée voire opprimée par la majorité anglophone ; une minorité qui commence à peine, dans les années $50 \mathrm{du} \mathrm{XX}^{\mathrm{e}}$ siècle, à revendiquer ses droits.

En plus, « le théâtre contemporain est l'un des moyens privilégiés de l'expression des minorités ethnoculturelles. Il dénonce souvent les injustices sociales et les discriminations, et, plus généralement, facilite la mise au jour des questions débattues dans la communauté $\gg^{2}$ constate Madelena Gonzalez dans l'avant-propos des actes du colloque sur les minorités au théâtre tenu à Avignon en décembre 2006. Rappelons que le théâtre québécois est relativement jeune ${ }^{3}$ et lui-même, il fait partie d'un art minoritaire. La dramaturgie de Marcel Dubé s'inscrit donc parfaitement, à mon sens, dans l'optique du théâtre minoritaire : premièrement par l'auteur luimême et, deuxièmement, par le contenu de son œuvre. Et mon propos sera d'examiner les différents aspects de la situation compliquée de la minorité québécoise au Canada dans les années $50 \mathrm{du} \mathrm{XX}^{\mathrm{e}}$ siècle : comment être une minorité ?

1 Interview accordée à Montréal, le 27 mars 1972.

2 P. Brasseur et M. Gonzalez (éds.), Théâtre des minorités. Mises en scène de la marge à l'époque contemporaine, Paris, Harmattan, 2008, p. 10.

3 Dans l'historiographie théâtrale québécoise on peut trouver trois dates différentes de la naissance du théâtre québécois. Premièrement, 1948 et la publication de Tit-Coq de Gratien Gélinas, ensuite 1958 et la publication d'Un simple soldat de Marcel Dubé et, enfin, 1968 et la publication des Belles-Sœurs de Michel Tremblay. 
Il ne saurait être question ici d'évoquer toutes les perspectives ou les (re) lectures possibles des pièces soumises à l'examen. Bien plus simplement, il s'agira d'une approche quelque part personnelle, une sorte de relecture par moments, des pièces considérées comme de vrais jalons du théâtre national québécois, d'un auteur qui a contribué non seulement à la naissance mais aussi à l'émergence de la dramaturgie québécoise. Déjà en 1959, Jean-Paul Vanasse décrit la production du dramaturge de la façon suivante :

Lire le théâtre de Marcel Dubé, c'est entrer dans un monde de héros sans emploi, d'êtres rivés à leur médiocrité, malgré eux. Un monde d'hommes écrasés par la vie, emmurés dans la pauvreté, de petites gens qui vivent tristement des jours sans espoir. Un monde de personnes brimées par l'existence, qui aspirent à un destin moins terre à terre, moins borné, qui veulent ouvrir leurs horizons, acquérir à leurs propres yeux une certaine dignité ${ }^{4}$.

Et si l'on ajoute que Marcel Dubé vient de décéder (le 7 avril 2016), à l'âge de 86 ans, en laissant plus de 300 textes pour le théâtre, la radio et la télévision, on comprend mieux l'importance de cet auteur prolifique pour la confirmation de l'identité québécoise. Étant donné la problématique adoptée, j'ai résolu de me concentrer sur des protagonistes jeunes, adolescents parfois, qui, de par leur nature, sont des êtres révoltés et contestataires. Ainsi, vue par le prisme de leur perception du monde, je montrerai la condition dépourvue de la minorité francophone au Canada à l’aube de la Révolution tranquille.

\section{Aspect familial}

Les adolescents ou de jeunes adultes de toutes les trois pièces choisies dénoncent voire accusent l'assujettissement de leurs parents ; la soumission qui mène à la misère des familles entières. Or la situation familiale des protagonistes diffèrent d'une pièce à l'autre : ceux de Zone $e^{5}$ font partie d'une bande et vivent dans la clandestinité. En se mettant à la marge de la société, ce groupe des jeunes forme une minorité qui se révolte contre son sort, contre les parents ou, plus largement, contre le monde des adultes auquel ces adolescents ne veulent pas adhérer. Ils refusent d'hériter la vie que leurs parents ont menée, d'hériter aussi la pauvreté et l'asservissement de leurs proches. Tarzan est orphelin donc, pour lui, le problème des parents ne se pose pas. Au contraire, il cherche à se construire

4 J.-P. Vanasse, « Marcel Dubé ou les chemins sans issues », Liberté, vol. 1, n 6, 1959, p. 356, www.id.erudit.org/iderudit (page consultée le 11 janvier 2016).

5 M. Dubé, Zone, Ottawa, Leméac, 1968. Toutes les citations viennent de cette édition et seront signalées par le sigle $Z$ suivi des numéros des pages citées. 
une famille avec d'autres jeunes de la rue et, en même temps, à contester l'ordre traditionnel. Ciboulette, quant à elle, ne se sent pas aimée par ses parents qui souhaitent se débarrasser d'elle. À 16 ans, elle doit se débrouiller comme si ses parents étaient morts, autre sorte d'orphelinat.

Florence de la pièce éponyme, tout en aimant ses parents comme elle le constate suite à son aventure malheureuse avec Eddy : « J'ai découvert trop tard que je les aimais $\gg^{6}$, reproche à son père d'avoir eu peur tout au long de sa vie :

T’as pas réussi à être propriétaire de ta propre maison en trente ans. T'es toujours resté ce qui tu étais : un p'tit employé de Compagnie qui reçoit une augmentation de salaire tous les cinq ans. T’as rien donné à ta femme, t’as rien donné à tes enfants que le strict nécessaire. Jamais de plaisirs, jamais de joies en dehors de la vie de chaque jour. [...] la même vie que t'as eue les [les enfants] attendait. Ils se sont mariés à des filles de rien pour s'installer dans des maisons comme la nôtre, grises, pauvres, des maisons d'ennui. Et pour moi aussi ce sera la même chose si je me laisse faire. Mais je ne veux pas me laisser faire, tu comprends papa! (F, p. 84).

Elle accuse aussi la vie de sa mère qui n’a jamais rien revendiqué, qui est toujours restée silencieuse, soumise à son mari et ses enfants, qui n'a pas osé avoir des rêves et une vie à elle : « Je ne veux pas devenir une machine à faire des enfants, je ne veux pas devenir une machine à faire du ménage, une machine à engraisser et à vieillir. [...] Je veux mieux que ça, je veux plus que ça $\gg(F, p .82,84)$. En poursuivant ses rêves, elle décide de partir mais sa décision sème un germe de révolte chez ses proches qui se heurtent à la vie avec plus de courage (le père adhère au syndicat pour revendiquer les droits des ouvriers et Pierre, le frère, accepte le rôle dans Britannicus).

Joseph Latour, le simple soldat, fait partie d'une même minorité des jeunes révoltés, malheureux au sein de la famille. Il se situe au milieu entre Tarzan et Florence car il est orphelin de la mère mais il vit avec son père. Sans le nommer explicitement, l'indignation de Joseph vise surtout le destin, un être ou une force suprême qui décide du sort des hommes : «Y a quelqu'un qui a triché quelque part pis qui fait que la vie maltraite toujours les mêmes !Y a quelqu'un qui a mêlé les cartes, Émile, pis va falloir le trouver. Va falloir le battre à mort, Émile... Ça fait assez longtemps que je le cherche ! Je vas le trouver ! Je vas le trouver ! $\gg^{7} \mathrm{Ce}$ pendant, il demeure seul dans sa protestation, son cri n'est entendu de personne et ne fait aucun effet sur son entourage. Émile, son ami, se résigne finalement en acceptant un travail médiocre et Édouard, son père, après avoir avoué s'être trom-

6 M. Dubé, Florence, Ottawa, Leméac, 1970, p. 137. Les citations qui suivront seront signalées par le sigle $\mathrm{F}$ suivi de la pagination.

7 M. Dubé, Un simple soldat, Ottawa, Institut Littéraire du Québec Ltée, 1958, p. 289. Pour les citations suivantes, on utilisera le sigle SS suivi de la pagination. 
pé et gâché sa vie, meurt dans une profonde solitude. Seule Fleurette, la petite sœur et amie de Joseph, aura le courage de s'opposer à sa mère, « la grosse Bertha », et d'affronter la vie avec plus de confiance. Mais après le départ de Joseph et la disparition du père, elle ne trouve plus aucun appui auprès de ses proches qui souhaitent se débarrasser d'elle.

Tous les adolescents des trois pièces soumises à l'analyse sont issus d'un milieu prolétaire où les parents ont du mal à joindre les deux bouts et pour lesquels les enfants constituent une charge importante. C'est pourquoi ces enfants doivent se mettre à travailler très tôt et, même s'ils restent au foyer, ils doivent contribuer financièrement à la vie de la famille. Cette situation provoque qu' ils se sentent solitaires car la famille ne les protège plus. C'est donc en solitude que les jeunes personnages de Dubé doivent affronter l'hostilité et l'injustice sociales.

\section{Aspect social}

Se considérant comme un auteur engagé, Dubé s'identifie entièrement avec la société canadienne-française à laquelle il appartient, sa société, qui deviendra bientôt une société québécoise. Tous les jeunes personnages de ses pièces ${ }^{8}$ sont nés de prolétaires peu instruits qui se soumettent à leur destin et acceptent la loi de plus fort :

Les personnages de Dubé étaient les victimes d'une fatalité dont on pouvait pour une bonne part imputer la responsabilité à la société à laquelle ils appartenaient et qu'ils acceptaient néanmoins, faute de percevoir ses assises idéologiques, à cause de cette ambiguiité qui leur faisait en même temps attaquer un ordre de choses sans en contester les principes?.

Les protagonistes de Zone se mettent hors la société et, ce qui s'ensuit, se placent hors la loi officielle. Ils vivent dans une sorte de rêve que l'auteur appelle le jeu ; l'illusion qu'ils se font de la vie en société. Le jeu, c'est la liberté, le sentiment de l'indépendance que la société reprend quand l'individu s'y intègre et s'y soumet en acceptant ses règles. Mais cette même société ne tolère pas ceux qui s'y opposent et leur impose un procès pour les accuser d'avoir violé les lois sociales et les condamner. Vient donc ce qui est inévitable, la mort ; mais cette mort est tragique car elle advient beaucoup trop tôt en coupant ainsi les liens fragiles qui unissent la petite bande des contrebandiers de cigarettes. Leur désir est ordinaire, même banal : fuir leur milieu défavorisé, se faire un peu d'argent pour commencer enfin une vie heureuse et oublier une enfance triste et pitoyable

${ }^{8}$ Les pièces écrites jusqu'à la fin des années 50 car, à partir des années 60, Dubé passe de la période de l'adolescence à la période bourgeoise.

9 M. Laroche, Marcel Dubé, Montréal, Fides, 1970, p. 53. 
dans un quartier pauvre. Selon Madeleine Greffard, on peut y observer « pour la première fois le Canadien français confronté véritablement avec une société qui lui est étrangère et hostile $\gg^{10}$.

Quant à Florence, genre d'Antigone québécoise, elle est aussi lucide que son ancêtre antique. Son aspiration à la liberté, son refus de la vie sombre et plate de ses parents, sa conscience de l'injustice sociale impressionnent le lecteur/spectateur et font preuve d'une dignité caractéristique pour des êtres purs et innocents ${ }^{11}$. Florence crie à ses parents qu'elle rêve d'une vie meilleure que la leur où il y aurait plus de liberté et d'égalité : « Je ne veux pas d'un homme qui se laissera bafouer toute sa vie, qui ne fera jamais de progrès [...]. Je préfère mourir plutôt que de vivre en esclavage toute ma vie » (F, p. 84-85). Mais c'est par le biais de son père Gaston que nous pouvons mieux connaître la situation sociale de la minorité francophone :

Sur les bancs de l'école, Toinette, à l'église le dimanche, aux campagnes électorales, dans les usines, dans les bureaux, partout, on nous a appris à avoir peur. [...] On nous a appris à avoir peur des fantômes pendant qu'on nous dépouillait de nos vrais biens. C'est comme ça qu'on nous a éduqués, c'est comme ça que les politiciens continuent de nous éduquer encore, c'est pour ça qu'on est devenu des poules mouillées. Je comprends Florence de ne pas vouloir partager sa vie avec une poule mouillée (F, p. 107).

Le choix de Florence est aussi difficile que celui d'Antigone car elle décide de quitter sa société et partir à New York pour essayer de trouver son bonheur.

Il en est de même pour Joseph Latour qui choisit l'uniforme de l'armée en se distanciant ainsi de la vie morne de son père et de l'hypocrisie de sa belle-mère et ses enfants : « Moi, j'aime l'action. [...] Si je reste à Montréal, je me connais, je vas retourner chez nous pis la même maudite vie qu'avant va recommencer $\gg$ (SS, p. 256). Joseph veut « devenir quelqu'un » au sein de la société qui laisse peu de place aux individus comme lui. Dans son discours, on retrouve des reproches ou des accusations adressées surtout à son père, qui lui-même avait du mal à trouver sa place dans la société. N’ayant pas confiance en lui, le père épouse « la grosse Bertha » que Joseph n’a jamais acceptée : « Ça fait vingt ans que tu couches avec elle pis que tu l'aimes pas... Tu l'as mariée parce que t’étais pas capable de rester tout seul, parce que t'étais lâche... [...] Mais fait attention, le père ! Moi, je suis là ! Je suis là pour te le faire regretter toute ta vie ! » (SS, p. 294) Ainsi Joseph et Édouard deviennent les représentants typiques de la minorité francophone du

${ }^{10}$ M. Greffard, « Théâtre », Études littéraires, vol. 2, n 2, août 1969, p. 225, www. id.erudit.org (page consultée le 11 janvier 2016).

11 Il convient de rappeler que la création de Marcel Dubé est souvent comparée à celle de Jean Anouilh dont Antigone a eu un impact considérable sur le jeune auteur québécois. 
Québec à la sortie de la Seconde Guerre mondiale : issue du milieu agricole et peu à l'aise dans le monde urbain dominé par les anglophones, la vieille génération est soumise et résignée tandis que la nouvelle - celle qui est déjà née en ville - se révolte et aspire à un meilleur avenir. Leur exemple montre aussi la situation sociale difficile du Québec dominé par la puissance de l'État et le pouvoir de l'Église. Joseph se sent solitaire au sein d'une société qui n'a pas besoin de lui : $\ll[. .$.$] j'ai pas d'avenir, j'ai pas de connection { }^{12}$, j'ai pas de protection nulle part ! Je suis un bon-à-rien, un soldat manqué qui a seulement pas eu la chance d'aller crever au front comme un homme... » (SS, p. 293)

Dans l'ensemble, il est à souligner que Dubé cherche à brosser un portrait de la condition sociale des Québécois, appelés encore dans les années 50 du XX siècle des Canadiens français. Il le montre par le prisme de la révolte des adolescents qui, à cette époque-là, lui sont les plus proches. Néanmoins, dans les pièces du dramaturge québécois, on n'assiste pas à une simple révolte des jeunes liée souvent au conflit de générations, mais on observe une révolte qui est un cri au secours articulé par une minorité francophone au Canada.

\section{Aspect éducatif}

Ignorés par la famille, défavorisés par la société, les jeunes francophones n'ont pas les mêmes possibilités de s'instruire que leurs homologues anglophones. De toute façon, rares sont les cas où ils peuvent se former à leur gré ${ }^{13}$. Premièrement, les parents eux-mêmes ne sont pas instruits et, de ce fait, n'insistent pas suffisamment sur l'éducation de leurs enfants et, deuxièmement, ils sont trop pauvres pour pouvoir financer les études de nombreux enfants ${ }^{14}$, qui, à leur tour, se voient obligés d'intégrer le monde professionnel très tôt et de contribuer à la vie au foyer familial. Telle est la situation de Ciboulette dans Zone, de Florence dans la pièce éponyme ou Fleurette, Marguerite et Armand dans Un simple soldat.

Du point de vue de l'éducation, Dubé a un penchant pour les filles qui regrettent plus de ne pas pouvoir suivre leur formation (Florence, Fleurette) tandis que les garçons, mis à part Pierre, semblent ne pas vouloir faire des efforts.

${ }^{12}$ La version originale de l'édition citée.

${ }_{13}$ Dans les pièces étudiées, il n'y en a que deux : Pierre dans Florence et Ronald dans Un simple soldat. Issu d'une famille bourgeoise (son père s'est enrichi pendant la guerre en fabriquant des munitions), l'étudiant en sciences, Ronald annonce déjà l'étape suivante de la production de Marcel Dubé, celle où il peint la bourgeoisie québécoise.

${ }^{14}$ Rappelons le fameux phénomène de la « Revanche des berceaux » qui a duré au Canada francophone depuis la déportation des Acadiens en 1755 jusqu’à la Révolution tranquille dans les années $60 \mathrm{du} \mathrm{XX}$ sicècle. Durant cette période, une femme française avait, en moyenne, plus de 10 enfants. 
Fleurette dans son dialogue avec Joseph est vraiment émouvante : elle rêve d'être cultivée tout de suite pour mieux comprendre son petit copain Ronald : « C'est tout de suite, Joseph... tout de suite que je voudrais être instruite ! (SS, p. 277) Et ce dernier promet de lui financer l'université comme s'il lui promettait la lune... Quant à Florence, elle regrette aussi de ne pas pouvoir continuer son éducation, et reproche à ses parents d'avoir donné la possibilité d'étudier uniquement à Pierre, le plus petit : «Seulement Pierre qui a eu la chance de s'instruire : c'est lui qui le méritait le moins. Les autres, après la petite école, c'était le travail $\gg(\mathrm{F}, \mathrm{p} .84)^{15}$. À l'instar de Fleurette, elle est attirée par le beau monde des gens cultivés et elle aimerait mieux comprendre son patron, Eddy.

Pour ce qui est de Joseph, la situation est plus compliquée : «Je sais rien faire, j'ai jamais rien appris! La « business », les chiffres, le commerce, je connais rien là-dedans. Tout ce que j'ai, je te le répète, c'est mes deux mains. Rien que ça, le père, pas d'autres choses... » s'écrit-il à son père (SS, p. 229). Toutefois, dans le cas de Joseph, ce n'est pas la faute du père qui a tout essayé pour que son fils aille à l'école. Au début, Joseph était bon en classe, mais la perte subite de la mère a provoqué en lui un vif sentiment de révolte contre l'injustice du destin « Et puis, tout à coup, crac! en quatrième année, quand sa mère est morte, il est arrivé le dernier de sa classe dix fois sur dix. L'année suivante, il a refusé d'y retourner ; [...] » (SS, p. 286). Armand et Marguerite ne sont pas mieux éduqués que lui et quand sa fille déclare à Bertha qu'elle travaille comme secrétaire, cette dernière s'étonne : «J'ai toujours pensé que ça prenait de l'instruction pour devenir secrétaire » (SS, p. 219). Cette constatation semble être le meilleur exemple du niveau très bas des personnages dubéens.

\section{Aspect professionnel}

Le chômage ou de petits emplois sans avenir : voilà le choix que les jeunes des pièces de Dubé affrontent à la sortie de leur enfance. Issus des familles pauvres, sans appui des parents, sans instruction quelconque, ils sont condamnés à un travail d'esclave ou bien ils peuvent se mettre à la marge de leur société (chômeurs ou trafic illégal). Tel est le cas des contrebandiers de Zone qui essaient de s'enrichir très vite. Ainsi, on peut voir Moineau, un jeune de 20 ans, qui ne trouve que de petits boulots temporaires : « Toutes sortes de choses. Un jour j'en fais une, le lendemain j'en fais une autre $[. .$.$] je fais jamais la même chose \gg(Z, p .84)$. Quant à Ciboulette, elle présente sa situation de la façon suivante : « Mes parents sont pauvres et m'aiment pas beaucoup, mais ça m'est égal, j'attends pas après eux autres pour me faire vivre. J'ai

15 Dans cette famille, fréquenter le collège est un rêve qui donne accès au bonheur : «Quand tu auras terminé tes études, tes diplômes t'ouvriront toutes les portes. [...] T'auras ce qu'il faut pour devenir un grand homme $\gg(\mathrm{F}, \mathrm{p} .111)$. 
seize ans, je travaille à la manufacture et ça me fait rien parce qu'en même temps je suis contrebandière $[. ..] \gg(Z$, p. 49). Mais c'est grâce à Tarzan, le chef de la bande, que nous pouvons connaître la détermination du groupe : «Personne pourra nous obliger à travailler et à nous salir comme des esclaves dans des usines ou des manufactures. On gaspillera pas notre vie comme les autres gars de la rue qui se laissent exploiter par n'importe qui » (Z, p. 63). Les membres de ce groupe choisissent une voie fragile qui balance à la zone du danger mortel, mais c'est le présent qui compte. Même s'ils rêvent d'un meilleur avenir, ils ne pensent pas au demain, leur devise serait la fameuse maxime carpe diem.

Au niveau professionnel, Florence est placé entre deux mondes contradictoires, représentés par Eddy, son patron qui mène une vie insouciante et libertine, et Maurice, son fiancé, un homme sérieux, fidèle, sur lequel elle peut toujours compter. Mais le monde de Maurice est gris, plat, monotone, ennuyeux et même repoussant, tandis que celui d'Eddy attire la jeune fille malgré elle. Son poste de secrétaire lui permet de découvrir « la vie en couleurs » et elle ne veut plus voir que $\ll$ du noir et du blanc $\gg$.

Quant à Joseph, il est le type de chômeur qui ne réussit jamais à garder une place. Il rêve du service militaire pour se prouver qu'il vaut quelque chose : « Soldat, c'est la meilleure $\ll$ job $»$. [... Avant que je m'enrôle [...] j'étais pas capable de garder une $\ll$ job $»$, j'en voulais à tout le monde, je faisais mal mon travail, je me battais avec mes « foremen ». J'étais jamais content... » explique-t-il à son père (SS, p. 207). Les enfants de Bertha, Armand et Marguerite, n'ont pas plus d'instruction que Joseph mais ils ont choisi des voies différentes : Fleurette, la petite, travaille « à la manufacture », Armand après avoir suivi une formation travaille dans l'assurance et Marguerite fait commerce de ses charmes corporels dans la rue en travaillant pour Ti-Mine - seul personnage francophone qui gagne bien sa vie mais de façon malhonnête. En général, les francophones dans les pièces de Dubé des années 50 du $\mathrm{XX}^{\mathrm{e}}$ siècle ne deviennent pas responsables, chefs dans les usines, en dépendant toujours des plus forts car ils travaillent dans des $\ll$ maudites compagnies d'Anglais $\gg$.

\section{Aspect linguistique}

Placé hors du milieu universitaire ou théâtral ${ }^{16}$, Dubé se concentre sur des problèmes de la langue, de même que sur ceux de l'esthétique littéraire : «Je ne me noierai pas dans le melting pot qu'on nous propose, je me suiciderai plutôt à sa

16 Marcel Dubé a suivi l'enseignement universitaire classique et n'a jamais reçu une éducation théâtrale proprement dite, mis à part un séjour de formation en France, en 1953, pendant lequel il fréquente les écoles d'art dramatique de Paris en tant que boursier de l'État. Par contre, il a volontiers collaboré avec les professionnels de l'art de Melpomène et a écrit certaines pièces pour des acteurs précis, par ex. Florence pour Monique Miller. 
périphérie $\gg$ avoue-t-il lors d'une conférence ${ }^{17}$. De cette façon, il fige sa place intermédiaire dans l'histoire de la dramaturgie québécoise et prépare le terrain pour une vague des pièces écrites en langue populaire, appelée le joual, la vague entamée en 1968 par Michel Tremblay et la publication des Belles-Sœurs.

Dans les pièces de Dubé, le français est la langue que l'on utilise à la maison, entre les proches, dans les situations non officielles, tandis que l'anglais est la langue de travail et des lieux publics. Déjà dans Zone, quand Tit-Noir est interrogé par la police, il déclare : « [...] je comprends pas la langue que vous me parlez. Pour moi c'est comme de l'anglais, ou du chinois » (Z, p. 94). Il convient de signaler que dans cette première pièce, le dramaturge n'insère pas encore de mots anglais pour différencier les deux mondes opposés. Par contre dans Florence, on retrouve des passages en anglais qui marquent bien cette opposition. Ainsi, Florence, qui est vraiment un lien entre deux mondes, répond au téléphone en anglais : «William Miller Advertising !... Just a moment, please... » (F, p. 132) Eddy et son amie Madeleine, mannequin du milieu publicitaire, parlent français mais, en présence de quelqu'un d'autre au bureau, ils se mettent à parler anglais :

Eddy : « How's my sweetheart? »

Madeleine : «Like a brand new one my dear ».

Eddy : «Hello, mon chou ! »

Madeleine : « Toujours aussi séduisant, tu ne vieillis pas » (F, p. 45).

Néanmoins, c'est dans Un simple soldat que Dubé utilise « un langage naturel, c'est-à-dire conforme à la vérité de ses personnages et de leur milieu $\gg^{18}$, un langage choquant pour certains critiques (puristes ?) qui le traitent d' « ordinaire $\gg$, , bas $\gg, ~ «$ trivial $\gg, ~ «$ farci d'anglicismes $\gg$, surtout au moment de la publication de la pièce ${ }^{19}$. La langue que les personnages de cette pièce parlent au quotidien est déformée par l'emprise de l'anglais prédominant en ville, dans un

17 Conférence prononcée par Marcel Dubé en 1968 pendant un colloque sur le théâtre au Festival d'été de Sainte-Agathe sur les problèmes de la langue qui se posaient au dramaturge canadien-français. Et dans une interview accordée à Aurélien Boivin et André Gaulin en 1980, Marcel Dubé constate : « La langue française est devenue indissociable de ma survie comme Québécois et comme écrivain. S'il est vrai que nous formons un peuple qui n'est plus né pour un p'tit pain, je crois de la même façon que nous ne sommes pas nés pour la langue d'un p'tit joual. Les Québécois ne peuvent plus se permettre de s'amuser avec un p'tit joual, cela fait trop l'affaire des anglophones », Québec français, octobre 1980, p. 35.

18 N. Leroux, « III. Le théâtre », Études françaises, vol. 13, n 3-4, 1977, p. 344, www.id.erudit.org (page consultée le 11 janvier 2016).

${ }_{19}$ Cité d'après J. Przychodzeń, « Marcel Dubé, auteur « tragique » », Jeu, no 106, 2003 (1), p. 90. 
milieu industriel. Mais en même temps, c'est la langue qui est familière au public auquel cette pièce est adressée. Ils la comprennent bien même si c'est une sorte d'amalgame du français et de l'anglais.

\section{Conclusion}

Le théâtre de Marcel Dubé est une peinture symbolique de la collectivité québécoise à un moment historique bien déterminé, à savoir les années après la Seconde guerre mondiale et avant la Révolution tranquille ${ }^{20}$. Une période particulièrement difficile pour les jeunes gens dont les parents se mettent à quitter la campagne et s'installent en ville. Ils y sont aussi perdus que leurs géniteurs, placés sous la domination de vieux urbains, anglophones. Les familles presqu'aussi nombreuses qu'en milieu agricole n'ont pas la possibilité de leur assurer une formation convenable qui permettrait d'aspirer à un meilleur avenir. Dans Zone même le chef de police, qui a un fils du même âge que Tarzan et dont le rôle est d'accuser l'assassin, ne condamne pas le chef de la bande et évite de l'appeler meurtrier : «C'est surtout un pauvre être qu'on a voulu étouffer un jour et qui s'est révolté... Il a voulu sortir d'une certaine zone de la société où le bonheur humain est presque impossible $\gg$ (Z, p. 138).

Sans aucun doute, l'image de la société québécoise - ou encore canadienne-française selon l'auteur lui-même - présentée par Marcel Dubé dans ses premières pièces dépeint une certaine réalité historique du Canada francophone. Néanmoins, il serait simpliste de constater qu'elles sont uniquement une sorte de témoignage de l'époque car on y trouve aussi - ou surtout - la condition d'un jeune être humain, révolté, en quête de sa propre identité : identité nationale mais également linguistique, historique, familiale etc.

Issus d'un milieu pauvre, les protagonistes des pièces analysées se révoltent contre leur condition sociale. Or, il est intéressant de souligner que les pièces bourgeoises de Dubé, caractéristiques pour la décennie suivante, mettent en scène les mêmes jeunes gens révoltés contre les parents et la société injuste, même si les motifs de cette révolte ne sont plus d'une même nature.

${ }^{20}$ Je pense ici aux pièces qui constituent l'objet de cette étude car, bien évidemment, le dramaturge continue d'écrire et, dans les œuvres suivantes, il se focalise sur d'autres types de protagonistes et d'autres milieux sociaux. 


\section{Disadvantaged francophone minority in the theater of Marcel Dubé Zone (1953), Florence (1957), An ordinary soldier (1958)}

A major figure in the Quebec Theater, Marcel Dubé belongs to the generation of the playwrights that introduces the francophone theater to French Canada. Born in 1930, contemporaneous with Michel Tremblay, Jean Barbeau, Antonine Maillet and Michel Garneau, becomes known to the public at large by his play Zone, written in 1953, in a period of a "great political and economic darkness". Subsequently, Florence from 1957 and An ordinary soldier from 1958, depict a minority of young disadvantaged people; a francophone minority facing all powerful Anglophones. The main characters of these three plays as representatives of an entire generation in the history of Quebec, serve us as an example in order to show a certain reality of francophone Canada.

Keywords: Quebec theater - Marcel Dubé - rebellion - French minority Mots-clés : théâtre québécois - Marcel Dubé - révolte - minorité francophone 